\title{
A highly linear gilbert cell OTA with multiple gated transistors for non-coherent UWB receivers
}

\author{
Mostafa Yargholi ${ }^{\text {a) }}$ and Abdolreza Nabavi \\ Department of Electrical Engineering, Tarbiat Modares University (TMU), \\ PO Box 1411713116, Tehran, Iran \\ a)yargholi@modares.ac.ir
}

Abstract: This paper presents a highly linear Gilbert cell OTA by cancelling the second order transconductance derivative $\left(\mathrm{gm}^{\prime \prime}\right)$ of nMOS transistors near threshold voltage using the technique of multiple gated transistors. This gives rise to $5 \mathrm{~dB}$ improvement in IIP3 of OTA, using a $0.13 \mu \mathrm{m}$ CMOS technology with $1.2 \mathrm{~V}$ supply. Simulation results and analytical relations for THD and IIP3 in OTA illustrate the tradeoffs between linearity, bandwidth, and power dissipation. System level simulation of a non-coherent UWB receiver, with this OTA as a $\mathrm{Gm}-\mathrm{C}$ integrator in receiver structure, illustrates an improvement of BER from $3 \mathrm{e}^{-2}$ to $1 \mathrm{e}^{-3}$. The power consumption, IIP3, and transconductance in OTA are $0.62 \mathrm{~mW}, 20.5 \mathrm{dBm}$, and $156 \mu \mathrm{A} / \mathrm{V}$, respectively.

Keywords: CMOS OTA, linearization, multiple gated transistors

Classification: Integrated circuits

\section{References}

[1] M. Yargholi and A. Nabavi, "Analog front end modules design in Noncoherent UWB receivers for sensor networks," IEEE Conference on semiconductor electronic (ICSE2008), Malaysia, pp. 64-68, Dec. 2008.

[2] W. Ghabrial and L. R. Carley, "Transconductance-C filter design for wireless telecommunications receivers," P.h.D Thesis, Electrical and computer engineering, Carnegie Mellon university, 2003.

[3] A. N. Mohieldin, E. Sánchez-Sinencio, and J. Silva-Martínez, "A LowVoltage Fully Balanced OTA with Common Mode Feedforward and Inherent Common Mode Feedback Detector," ESSCIRC, Texas A\&M University, USA, pp. 191-194, 2002.

[4] A. N. Mohieldin, E. Sánchez-Sinencio, and J. Silva-Martínez, "Nonlinear effects in pseudo differential OTAs with CMFB," IEEE Trans. Circuits Syst. Analog Digit. Signal Process., vol. 50, no. 10, pp. 762-770, Oct. 2003.

[5] B. Kim, J. S. Ko, and K. Lee, "A new linearization technique for MOSFET RF amplifier using multiple gated transistors," IEEE Microw. Guided Wave Lett., pp. 371-373, Sept. 2000. 


\section{Introduction}

Non-coherent receivers in wireless sensor networks require high performance modules with low complexity and low power consumption. Among these modules is the integrator block which needs an OTA with linearity and power consumption around $20 \mathrm{dBm}$ and $1 \mathrm{~mW}$, respectively [1]. Considering these requirements, Gilbert cell OTA appears to be superior compared to fully differential and pseudo-differential OTA structures [2]. Nevertheless, Gilbert cell requires a simple low-power technique to meet the above linearity performance.

This paper deals with the design of highly linear Gilbert cell OTA, using the technique of multiple gated transistors to cancel the second order transconductance derivative $\left(\mathrm{gm}^{\prime \prime}\right)$ for linearity improvement.

\section{Gilbert cell OTA nonlinearity}

In Gilbert OTA shown in Fig. 1, two cross-coupled differential pairs of MOS transistors in saturation are used to cancel the common-mode input voltage dependency and to make the transconductace $\left(\mathrm{G}_{\mathrm{m}}\right)$ tunable. The transconductance of differential pair is:

$$
\mathrm{G} m=\frac{\partial I_{\text {out }}}{\partial v_{\text {id }}}=\mu_{\mathrm{n}} \cdot c_{o x}\left(\frac{W}{L}\right)\left(V_{C M}-V_{T h}\right)
$$

where $\mathrm{V}_{\mathrm{CM}}$ is the common-mode input voltage, $\mathrm{V}_{\mathrm{Th}}$ is transistor threshold voltage, $\mu_{\mathrm{n}}$ represents the mobility, $\mathrm{C}_{\mathrm{ox}}$ denotes the oxide capacitance per unit channel area and $(\mathrm{W} / \mathrm{L})$ is transistor size.

The output transconductance of Gilbert cell is given by:

$$
\mathrm{G}_{\mathrm{m}}=\frac{\partial\left[\left(\mathrm{I}_{\mathrm{D} 1}+\mathrm{I}_{\mathrm{D} 3}\right)-\left(\mathrm{I}_{\mathrm{D} 2}+\mathrm{I}_{\mathrm{D} 4}\right)\right]}{\partial v_{i d}}=\mu_{\mathrm{n}} \cdot \mathrm{C}_{\mathrm{ox}}(\mathrm{W} / \mathrm{L}) \cdot \mathrm{V}_{\mathrm{b}}
$$

The value of $G_{m}$ is controlled through the biasing voltage $V_{b}$ (see Fig. 1), which also cancels the effect of $\mathrm{V}_{\mathrm{CM}}$ on $\mathrm{G}_{\mathrm{m}}$.

Short channel effect is the main source for nonlinearity in Gilbert OTA structure. In a short channel device, the mobility is a function of critical electric field (Ec) [3]. Considering this, the current of MOS transistor in the saturation region is:

$$
\mathrm{I}_{\mathrm{d}}=\frac{1}{2} \mu_{0} \cdot c_{o x}\left(\frac{W}{L}\right)\left(V_{G S}-V_{T h}\right)^{2} \frac{1+\lambda V_{D S}}{1+\theta\left(V_{G S}-V_{T h}\right)}
$$

where $\mu_{\text {eff }}=\frac{\mu_{0}}{1+E / E_{c}}, \theta=\frac{1}{L E_{c}}+\theta_{0}$, L is the device channel length, $\lambda$ denotes the output impedance constant, $\mu_{0}$ is the low-field mobility, E represents the longitudinal electric field, and $\mathrm{E}_{\mathrm{C}}$ is the critical electric field [4]. Assume that: $\alpha=\frac{k \lambda}{1+\lambda V_{D C}}, \gamma=\frac{\theta}{1+\theta \cdot v}$, and $K=\frac{1}{2} \mu \cdot c_{o x}\left(\frac{W}{L}\right) \frac{1+\lambda V_{D C}}{1+\theta \cdot v}$, where $\mathrm{k}$ is small signal gain, $\mathrm{V}_{\mathrm{DC}}=\mathrm{V}_{\mathrm{DD}}-\mathrm{V}_{\mathrm{DS}}$ and $\nu=\mathrm{V}_{\mathrm{CM}}-\mathrm{V}_{\mathrm{Th}}$. By substituting these in (3):

$$
\mathrm{I}_{\mathrm{d}}=K\left(v+\frac{v_{i d}}{2}\right)^{2} \cdot\left(1-\alpha \frac{v_{i d}}{2}\right) \frac{1}{1+\gamma \frac{v_{i d}}{2}}
$$


By using Taylor series expansion in (4) and using this in Gilbert cell structure (Fig. 1 without auxiliary transistors), the output current of Gilbert OTA is:

$$
\begin{aligned}
\mathrm{I}_{\mathrm{out}}= & \left(\mathrm{I}_{\mathrm{D} 1}+\mathrm{I}_{\mathrm{D} 3}\right)-\left(\mathrm{I}_{\mathrm{D} 2}+\mathrm{I}_{\mathrm{D} 4}\right) \\
= & K \cdot\left\{2\left[\left(2 v-V_{b}\right)-\frac{(\alpha+\gamma)}{2}\left(v^{2}-\frac{\left(v-V_{b}\right)^{2}}{\left(1-V_{b}\right)}\right)\right] \cdot v_{i d}\right. \\
& +(0) v_{i d}^{2}+2\left[\frac{1}{8} \alpha \frac{V_{b}}{1-V_{b}}+\frac{1}{8} \gamma \cdot \frac{V_{b}}{1-V_{b}}+\frac{1}{4} \alpha \cdot \gamma \cdot\left(v-\frac{v-V_{b}}{\left(1-V_{b}\right)^{2}}\right)\right. \\
& \left.-\frac{1}{8} \alpha \cdot \gamma^{2} \cdot\left(v^{2}-\frac{\left(v-V_{b}\right)^{2}}{\left(1-V_{b}\right)^{3}}\right)+\frac{1}{4} \gamma^{2} \cdot\left(v-\frac{v-V_{b}}{\left(1-V_{b}\right)^{2}}\right)-\frac{1}{8} \gamma^{3} \cdot\left(v^{2}-\frac{\left(v-V_{b}\right)^{2}}{\left(1-V_{b}\right)^{3}}\right)\right] v_{i d}^{3} \\
& +(0) v_{i d}^{4}-\frac{1}{16} \gamma^{2} \cdot\left[(\alpha+\gamma)\left(1-\frac{1}{\left(1-V_{b}\right)^{3}}\right)-2 \alpha \cdot \gamma \cdot\left(v-\frac{v-V_{b}}{\left(1-V_{b}\right)^{4}}\right)\right. \\
& +\gamma^{2} \cdot \alpha\left(v^{2}-\frac{\left(v-V_{b}\right)^{2}}{\left(1-V_{b}\right)^{5}}\right)-2 \gamma^{2} \cdot\left(v-\frac{v-V_{b}}{\left(1-V_{b}\right)^{4}}\right) \\
& \left.\left.+\gamma^{3}\left(v^{2}-\frac{\left(v-V_{b}\right)^{2}}{\left(1-V_{b}\right)^{5}}\right)\right] v_{i d}^{5}+\ldots .\right\}
\end{aligned}
$$

Even harmonic distortions are canceled out in both differential and Gilbert OTA structures. Odd harmonic distortions by using $\nu_{\mathrm{id}}=\mathrm{V}_{\text {Peak }} \cdot \cos (\omega \mathrm{t})$ are given by:

$$
\begin{gathered}
H D_{3}(\text { Diff })=\frac{Z}{16} V_{\text {Peak }}^{2} \approx \frac{\theta}{4 v \cdot(1+\theta \cdot v)^{2} \cdot(2+\theta \cdot v)} V_{r m s}^{2} \\
H D_{5}(\text { Diff })=\frac{\gamma^{2} \cdot Z}{256} V_{\text {Peak }}^{4} \approx \frac{\theta^{3}}{32 \cdot v \cdot(1+\theta \cdot v)^{4} \cdot(2+\theta \cdot v)} V_{r m s}^{4} \\
H D_{3}(\text { Gilbert }) \approx \frac{Z^{\prime}}{16} V_{\text {Peak }}^{2} \approx \frac{\theta \cdot V_{b} \cdot\left[(1+\theta \cdot v)^{2}+2 \theta\right]}{4 v \cdot(1+\theta \cdot v)^{2} \cdot\left(2+2 \theta \cdot v-\theta \cdot V_{b}\right)} V_{r m s}^{2} \\
H D_{5}(\text { Gilbert }) \approx \frac{\gamma^{2} \cdot Z^{\prime \prime}}{256} V_{\text {Peak }}^{4} \approx \frac{\theta^{3}\left[\left(1-\frac{1}{\left(1-V_{b}\right)^{3}}\right)(1+\theta \cdot v)^{2}-2 \theta \cdot V_{b}\right]}{32 \cdot v \cdot(1+\theta \cdot v)^{4} \cdot\left(2+2 \theta \cdot v-\theta \cdot V_{b}\right)} V_{r m s}^{4} \\
\text { Where } Z=\frac{-\left(\frac{1}{v}-2 \gamma+\gamma^{2} \cdot v\right)(\alpha+\gamma)}{\left(1-\frac{\alpha+\gamma}{2} v\right)}, Z^{\prime}=\frac{-(\alpha+\gamma)\left(\frac{-V_{b}}{2 \cdot v}-2 \gamma \frac{V_{b}}{2 v}+\gamma^{2} \cdot V_{b}\right)}{\left(1-\frac{\alpha+\gamma}{2} \cdot V_{b}\right)} \\
\text { and } Z^{\prime \prime}=\frac{-(\alpha+\gamma)\left(\frac{\left(1-\frac{1}{\left(1-V_{b}\right)^{3}}\right)}{2 \cdot v}-2 \gamma \frac{V_{b}}{2 v}+\gamma^{2} \cdot V_{b}\right)}{\left(1-\frac{\alpha+\gamma}{2} \cdot V_{b}\right)} .
\end{gathered}
$$

Right sides of (6-9) are extracted by neglecting channel length modulation with $\alpha=0$ (for $\mathrm{k}=1$ and $\lambda<\theta$ ). Right sides of (8-9) are extracted by assuming that $\mathrm{V}_{\mathrm{b}} \ll \nu, \mathrm{V}_{\mathrm{b}} \ll 1,\left(1-\mathrm{V}_{\mathrm{b}}\right) \approx 1 \approx\left(1-\mathrm{V}_{\mathrm{b}}\right)^{2} \approx\left(1-\mathrm{V}_{\mathrm{b}}\right)^{3}$, and $\nu+\left(\nu-\mathrm{V}_{\mathrm{b}}\right) /\left(1-\mathrm{V}_{\mathrm{b}}\right)^{2} \approx 2 \nu-\mathrm{V}_{\mathrm{b}} \approx 2 \nu$. From these equations, we have $\mathrm{HD}_{3} \gg \mathrm{HD}_{5}$, so $\mathrm{THD} \approx \mathrm{HD}_{3}$. 
The IP3 of differential OTA is:

$$
\begin{aligned}
I P 3(\text { Diff }) & =\sqrt{\frac{4}{3} \cdot \frac{\alpha_{1}}{\alpha_{3}}}=\sqrt{\frac{4}{3} \cdot \frac{1}{H D_{3}}}=\sqrt{\frac{64}{3 Z \cdot V_{\text {Peak }}^{2}}} \\
& \approx \sqrt{\frac{16 v \cdot(1+\theta \cdot v)^{2} \cdot(2+\theta \cdot v)}{3 \theta \cdot V_{r m s}^{2}}}
\end{aligned}
$$

Also, the IP3 of Gilbert OTA is:

$$
I P 3(\text { Gilbert }) \approx \sqrt{\frac{64}{3 Z^{\prime} \cdot V_{\text {Peak }}^{2}}} \approx \sqrt{\frac{16 v \cdot(1+\theta \cdot v)^{2} \cdot\left(2+2 \theta \cdot v-\theta \cdot V_{b}\right)}{3 \theta \cdot V_{b} \cdot\left[(1+\theta \cdot v)^{2}+2 \theta\right] \cdot V_{r m s}^{2}}}
$$

These equations show the tradeoffs between linearity, frequency response and power consumption. In single MOS and differential pair, THD and power consumption are functions of $\nu$. For high linearity, we should decrease $\theta(\theta \alpha 1 / \mathrm{L})$, which at the same time increase the parasitic capacitances, leading to narrow band frequency response. Increasing $\nu$ also improves the linearity at the expense of extra power consumption.

By comparing IP3 in (10) and (11), it can be shown that $\mathrm{V}_{\mathrm{b}} \cdot\left[(1+\theta \cdot \nu)^{2}+\right.$ $2 \theta] \ll 1$; because $\mathrm{V}_{\mathrm{b}}<\nu \ll 1$ and $\theta<1$; consequently $\operatorname{IIP}_{3}$ (Gilbert) $\gg$ $\mathrm{IIP}_{3}$ (Diff). By comparing harmonic distortion of differential and Gilbert cell in (6) and (8), we conclude that $\mathrm{HD}_{3}$ (Diff) $\gg \mathrm{HD}_{3}$ (Gilbert). Therefore, Gilbert OTA can result in a relatively high linearity.

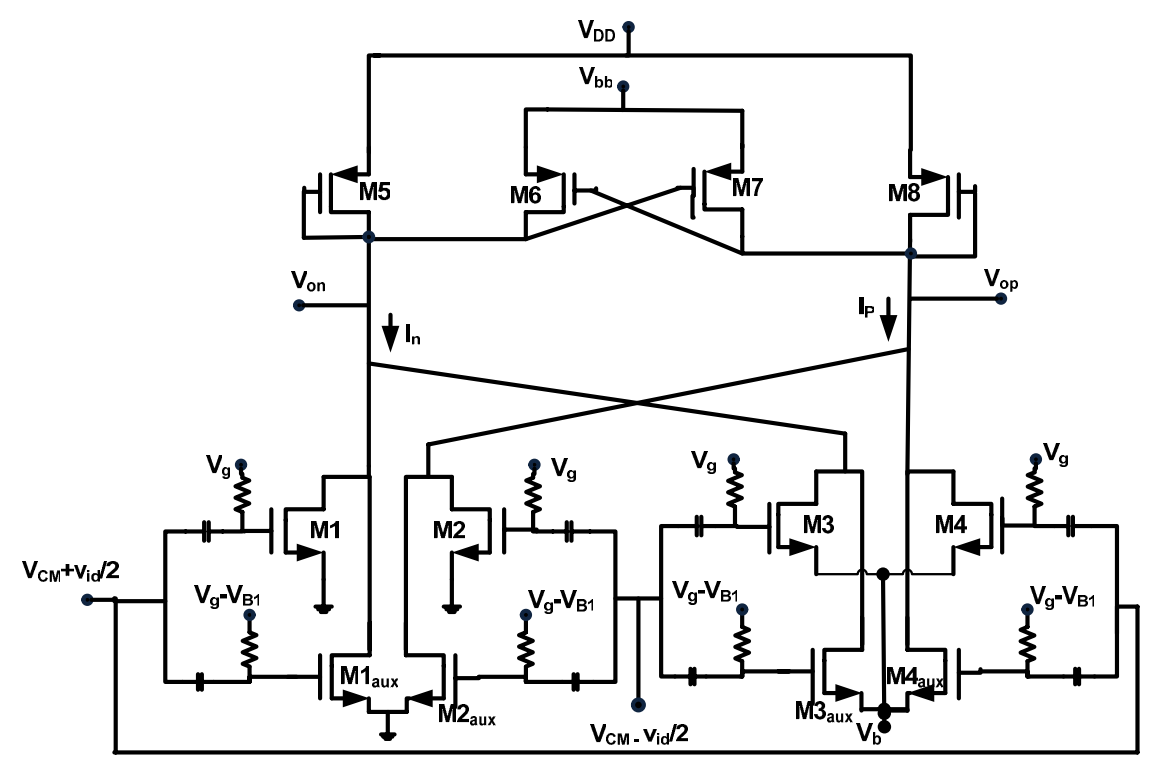

Fig. 1. Schematic of Gilbert cell OTA with one auxiliary transistor

\section{Proposed OTA with multiple gated transistors}

A simple low-power solution for linearity enhancement, without using onchip inductors, is the technique of multiple gated transistors [5]. In this technique, an auxiliary MOS is used to cancel the nonlinearity effect of the main MOSFET. 
The drain current of a common source MOS is expressed by:

$$
i_{d s}=g_{m} v_{g s}+\frac{g_{m}^{\prime}}{2 !} v_{g s}^{2}+\frac{g_{m}^{\prime \prime}}{3 !} v_{g s}^{3}+\cdots
$$

where $v_{g s}$ is gate-to-source voltage, $g_{m}^{(n)}$ denotes the $\mathrm{n}^{\text {th }}$ order $g_{m}$ derivative. To reduce the power consumption, $\left(\mathrm{vgs}_{\mathrm{gs}}-\mathrm{v}_{\mathrm{Th}}\right)$ is usually set near $0.1 \sim 0.2[5]$. In this bias region, $\mathrm{gm}^{\prime \prime}$ has a negative peak and degrade the linearity of OTA. Negative $\mathrm{gm}^{\prime \prime}$ of the main transistor is cancelled by positive $\mathrm{gm}^{\prime \prime}$ of auxiliary transistor. Main transistor is biased at $\mathrm{v}_{\mathrm{g}}$, but auxiliary transistor is biased at $\mathrm{v}_{\mathrm{g}}-\mathrm{v}_{\mathrm{B} 1}$ (Fig. 2. d), so the transfer characteristic curve for auxiliary transistor is shifted by $v_{B 1}$ as shown in Fig. 2. b. This figure shows the ADS simulation result with $0.13 \mu \mathrm{m}$ CMOS technology and for $\mathrm{v}_{\mathrm{B} 1}=0.15 \mathrm{~V}$. The main and auxiliary transistors are designed with the same size.

The schematic of highly linear multiple gated Gilbert cell OTA is shown in Fig. 1. In this figure, M1-M4 are biased in the saturation region to satisfy (2) for $G_{m}$. The sizes of M1-M4 are the same to cancel out the commonmode voltage effect at the output. The auxiliary transistors $\mathrm{M} 1_{\text {aux }}-\mathrm{M} 4_{\text {aux }}$ are biased in the region with positive $\mathrm{gm}^{\prime \prime}$ to cancel the negative $\mathrm{gm}^{\prime \prime}$ of the main transistor in OTA cell. PMOS transistors M5-M8 are used to provide
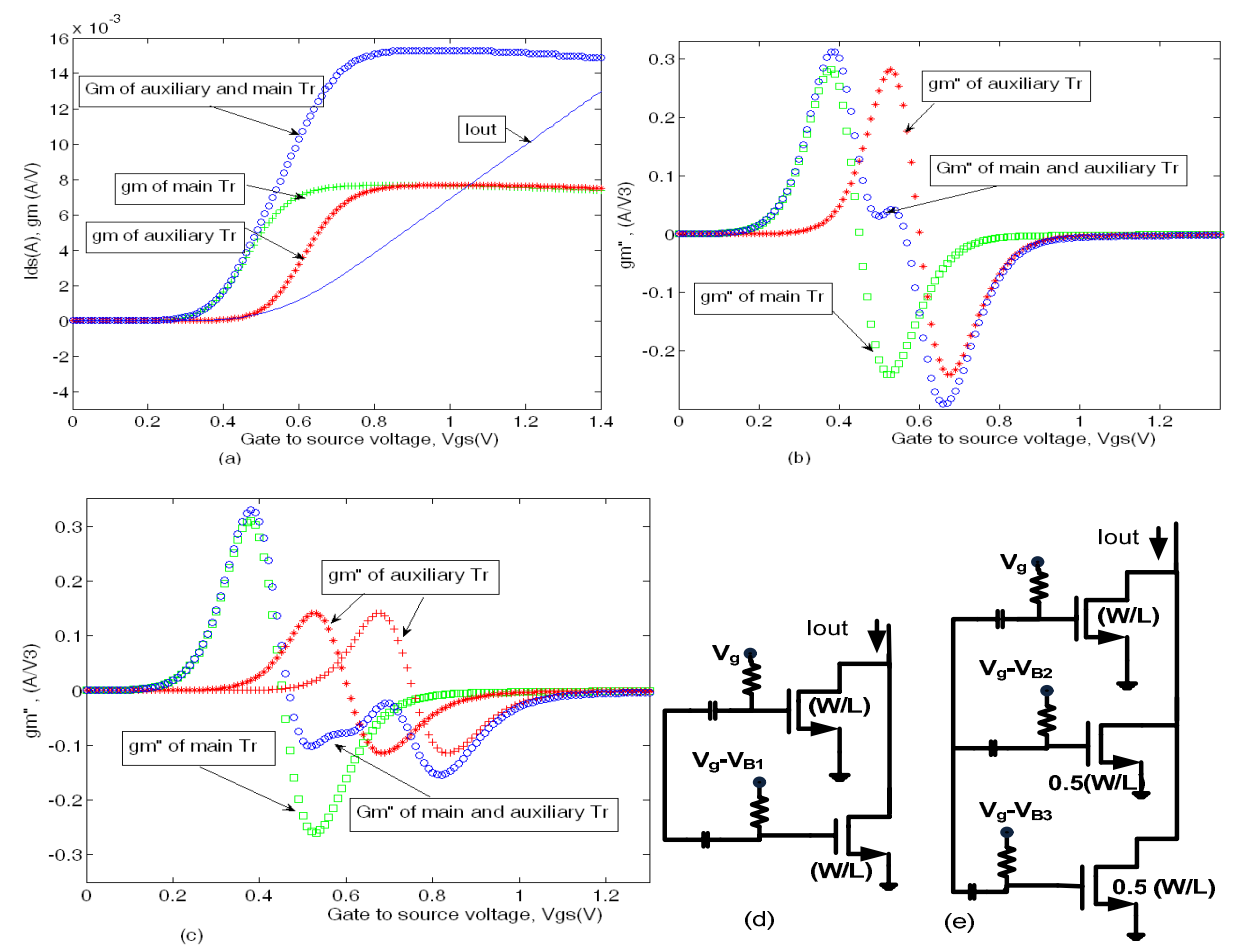

Fig. 2. Simulation results a) $g_{m}$ of main, auxiliary FET and their summation b) third-order nonlinear currents of main transistor, one auxiliary FET, and their summation c) decreasing sensitivity of ip3 to bias voltage, by using two auxiliary Transistors with $(\mathrm{W} / \mathrm{L})_{\text {aux }}=0.5(\mathrm{~W} / \mathrm{L})_{\text {main }}$ with $0.15 \mathrm{~V}$ and $0.3 \mathrm{~V}$ bias shift d) Multiple gated with one auxiliary Transistor e) Multiple gated with two auxiliary Transistors with half size 
OTA biasing current, and to form the active load at the output. The amount of bias voltage $\left(\mathrm{V}_{\mathrm{b}}\right)$ establishes a tradeoff between transconductance and linearity. A high value of $\mathrm{V}_{\mathrm{b}}$ gives high $\mathrm{G}_{\mathrm{m}}$, but low IP3 as shown in (10).

\section{Simulation results}

This section presents the results of Advanced Design System (ADS) simulator using a $0.13 \mu \mathrm{m}$ CMOS technology with $1.2 \mathrm{~V}$ supply. The IIP3 of OTA was obtained by two-tone test with frequency separation of $100 \mathrm{kHz}$ in $100 \mathrm{MHz}$. Harmonic balance (HB) and budget simulations were used for nonlinearity calculation. Simulation illustrates that by using multiple gated transistors in Gilbert OTA, the IIP3 is improved by $5 \mathrm{~dB}$, giving rise to $20.5 \mathrm{dBm}$ in $0.56 \mathrm{~V}$ bias voltage, as shown in Fig. 3 (a). This figure shows the IIP3 in OTA versus bias voltage before and after linearization. The value of $\theta$ is determined by a best fit to the simulated device characteristics for $0.13 \mu \mathrm{m}$ CMOS technology $\theta=0.35 \sim 0.4 \mathrm{v}^{-1}$. Analytical result for $\theta=0.38 \mathrm{v}^{-1}, \mathrm{~V}_{\mathrm{b}}=0.05$, and $\mathrm{v}_{\mathrm{Th}}=0.43 \mathrm{~V}$ is illustrated in Fig. 3. a. Therefore, by using the linearization technique we have $5 \mathrm{~dB}$ improvement in IIP3 for $\mathrm{V}_{\mathrm{g}}=0.56 \mathrm{~V}$.

As shown in Fig. 2 (b) and 3 (a), the IIP3 is sensitive to bias voltage. To decrease this sensitivity, we use two auxiliary transistors with half size and voltage shift of $0.15 \mathrm{~V}$ and $0.3 \mathrm{~V}$. As shown in Fig. 2 (c), $\mathrm{Gm}^{\prime \prime}$ summation of main and two auxiliary transistors is constant in wide range of bias voltage from $0.55 \mathrm{~V}$ to $0.64 \mathrm{~V}$, while IIP3 is $20.5 \mathrm{dBm}$ as shown in Fig. 3 (a). The power consumption of OTA with two auxiliary transistors is $0.62 \mathrm{~mW}$ for $0.56 \mathrm{~V}$ bias voltage as represented in Fig. 3 (b). Also, OTA has $156 \mu \mathrm{A} / \mathrm{V}$ transconductance while drawing only $0.516 \mathrm{~mA}$ from $1.2 \mathrm{~V}$ supply. Increasing $\mathrm{V}_{\mathrm{g}}$ also improves the transconductance at the expense of power consumption. In this case, $\mathrm{P}_{1-\mathrm{dB}}$ is equal to $10.18 \mathrm{dBm}$.

An Energy detection non-coherent UWB receiver modeled in IEE802.15.3.a with BPPM modulation. Transmitter composed of channel coding, source coding, modulation block, UWB pulse generator and power amplifier. Receiver consists of LNA, AGC, squarer module, integrator (I\&D) and synchronizer [1]. System level simulation of a non-coherent receiver in Simulink of MATLAB with OTA as a $\mathrm{Gm}-\mathrm{C}$ integrator improves BER of receiver from
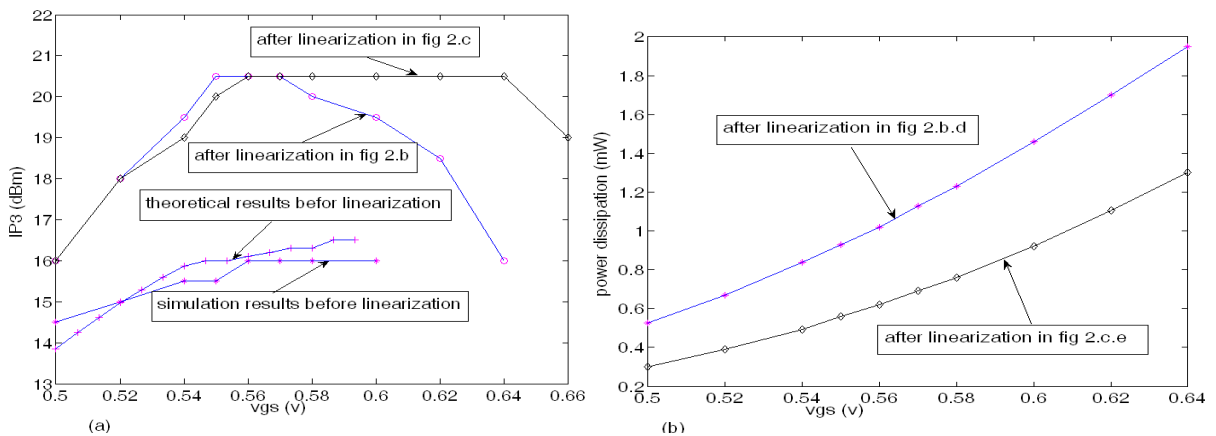

Fig. 3. Simulation result a) The IIP3 of Gilbert OTA, linearization performance and theoretical result b) power consumption $(\mathrm{mW})$ of proposed OTA 
$3 \mathrm{e}^{-2}$ to $1 \mathrm{e}^{-3}$.

\section{Conclusion}

Low-power linearization with multiple gated transistors was presented for a CMOS Gilbert OTA using a 0.13 um CMOS technology. In this linearization technique, negative $\mathrm{gm}^{\prime \prime}$ of main transistor is cancelled by positive $\mathrm{gm}^{\prime \prime}$ of auxiliary transistor. Simulations results illustrate $5 \mathrm{~dB}$ improvement of IIP3, giving rise to IIP3 of $20.5 \mathrm{dBm}$. The OTA dissipates $0.62 \mathrm{~mW}$, and $\mathrm{P}_{1-\mathrm{dB}}$ (in) is $10.18 \mathrm{dBm}$ for transconductance of $156 \mu \mathrm{A} / \mathrm{V}$. System level simulation of a non-coherent receiver with OTA as a Gm-C integrator illustrates improvement of BER from $3 \mathrm{e}^{-2}$ to $1 \mathrm{e}^{-3}$.

\section{Acknowledgments}

This work was supported by ITRC (Iran telecommunication research center). 